\title{
The overall assessment of The Medical Effects of Seat-Belt Legislation in the United Kingdom
}

\author{
J. G. AVERY \\ District Medical Officer, South Warwickshire, England
}

The study is an analysis of 14000 cases (car occupants) one year before and one year after legislation in the United Kingdom. It is not the largest study of its kind, but is the most comprehensive, especially in relation to injuries and their severity.

The law resulted in an overnight increase in the usage of front seat-belts from just under $40 \% \frac{3}{\mathrm{~N}}$. to around $95 \%$. Of course, if usage had gone from $0 \%$ to $95 \%$ then the implication of the results would be even stronger.

The original hypotheses for the study were:

(1) a reduction in the number of patients (out-patients, in-patients and deaths);

(2) a reduction in the number of bed-days for such patients;

(3) a reduction in the average severity of injuries;*

(4) a reduction in the number of severe injuries;

(5) a reduction in the multiplicity of both major and minor injuries to front-seat occupants;

(6) a low probability of death if injured;

(7) a reduction in brain injuries;

(8) a reduction in skull fractures; $*$

(9) a reduction in minor injuries to the face;

(10) a reduction in facial fractures;

(11) a reduction in injuries to the lungs;

(12) an increase in sprained necks;

(13) an increase in fractures of the sternum;

(14) back-seat passengers should not benefit to the same extent or follow the same pattern as those in the front seats;

(15) front-seat occupants who were belted will have lower mean severity scores in the first year than in the second;*

(16) front-seat occupants who were unbelted in the second year will have higher mean severity scores than in the first;

(17) late night and early morning front-seat occupants will have lower rates of belt wearing and higher mean severity scores than at other times of the day.

The report found 12 of the 17 hypotheses tested to be correct. One hypothesis and part of one hypothesis - both relating to death-could not be tested due to insufficient data. The hypotheses marked* could not be validated.

Overall the report found a $26 \%$ fall in fatalities for car occupants (based on the Coroner's study), a $15 \%$ fall in out-patient attendances and a $25 \%$ fall in patients admitted to hospital.

The study showed: The study showed:
There was a predicted fall in major injuries to lung and pleura in both drivers and front-seat
passengers.

- An overall fall in brain injury but a $44 \%$ increase (not statistically significant) in major brain injury to drivers. This is in contrast to similar studies in Sweden.

Correspondence: F. G. Avery, District Medical Officer, South Warwickshire, England. 
- A greater increase in 'sprained necks' for both passengers and drivers.

- The fatality study showed a $32 \%$ fall in the number of deaths in front-seat occupants and a $15 \%$ rise in deaths in rear-seat occupants. After legislation only $73 \%$ of front-seat occupants who died were wearing seat-belts, compared with a $95 \%$ wearing-rate in the community.

- There was a reduction in rib and clavicle fractures when other countries (e.g. Australia) have found a predictable increase. The increase in facial injuries in the UK is also at variance with the Australian findings.

- The greatest benefits were to the less seriously injured (in contrast to Swedish findings).

- Injuries to the eyes and spine were uncommon, but probably merit further study.

- Further enquiry is needed into the increase in major injury to the brain and the increase in fractures of the sternum, sprained necks and abdominal injury. This will require modification to seat belt design and car interior.

- The unbelted rear passenger may be a threat to the front-seat occupant and this needs to be explored and evaluated.

- A recommendation should be to lobby for the compulsory wearing of seat-belts in rear seats.

\section{POINTS RAISED IN OPEN DISCUSSION}

- There is some question as to whether belting rear-seat passengers significantly affects the outcome for front-seat passengers, and that impact by rear-seat passengers occurs very infrequently.

- It was the use of the AIS rather than the ICD which enabled fine details to be determined, in particular the effect on head injuries.

- The use of seat-belts has prevented many penetrating eye injuries. Belted front-seat occupants no longer hit the windscreen, but they may hit their heads on the fascia instead. 